\title{
Hospitalists, PCPs, Specialists, and Non-Physicians: Too Many Cooks in the Kitchen?
}

\author{
Daniel J. Brotman, MD, FACP, FHM and John R. Nelson²
}

${ }^{1}$ Hospitalist Program, Johns Hopkins Hospital, Baltimore, Maryland; ${ }^{2 H o s p i t a l i s t ~ P r a c t i c e, ~ O v e r l a k e ~ H o s p i t a l, ~ B e l l e v u e, ~ W a s h i n g t o n . ~}$

The tension between continuity of care and specialization is not new, but may have reached a tipping point when the hospitalist movement erupted onto the American medical scene in the late 1990s. By definition, when a hospitalist cares for an inpatient, there is some fragmentation of care, which is, at least in theory, avoidable - if the primary care provider (PCP) can serve as attending physician in the hospital. Literature has since emerged suggesting that clinical and economic outcomes of care by hospitalists are at least as good as that provided by PCPs, and that patients are not, in general, opposed to hospitalist care. ${ }^{1-3}$

However, the degree of discontinuity is not just a feature of whether a hospitalist assumes care of the hospitalized patient. Discontinuity can be exacerbated by changing attendings throughout the hospital stay. And inpatient continuity is a potential issue for both the hospitalist model and traditional model of care (in which the PCP serves as inpatient attending physician). While one might assume that the hospitalist model fosters more inpatient discontinuity because most hospitalists-whether working a 7-on-7-off schedule or another schedule-do not commit to caring for a patient throughout an entire hospitalization the way a PCP might, this question has not previously been examined. Even if the hospitalist model is a fait accompli in many hospitals, it is worth knowing how inpatient continuity differs between the 2 models.

In this issue of the Journal, Fletcher and colleagues ${ }^{4}$ used billing data to examine trends in inpatient continuity of care over a 10-year period ending in 2006, and sought to determine: (1) whether inpatient care has become more fragmented over time (as defined by the number of generalists caring for a patient over the course of an average hospitalization), and (2) whether inpatient care provided by hospitalists tends to be more fragmented than care provided by PCPs. They found that continuity of inpatient care has indeed

*Address for correspondence and reprint requests: Daniel J. Brotman, M D, FACP, FHM, Hospitalist Program, Johns Hopkins Hospital, 600 North Wolfe St/Park 307, Baltimore, MD 21287; Tel.: 443-287-3023; E-mail: brotman@jhmi.edu

Additional Supporting Information may be found in the online version of this article.

Received: April 20, 2011; Revised: June 17, 2011; Accepted: June 19, 2011

2011 Society of Hospital Medicine DOI 10.1002/jhm.960

Published online in Wiley Online Library (Wileyonlinelibrary.com). decreased over time. In 1996, just over $70 \%$ of patients received care from 1 generalist; this number declined to just under $60 \%$ a decade later, despite a decrease in length-of-stay during that period. However, and perhaps surprisingly, patients cared for exclusively by hospitalists saw fewer generalists in the hospital (ie, fewer different hospitalists) than those cared for exclusively by outpatient providers. The authors conclude that the doctor-patient continuity over the course of a hospital stay is not worse in the hospitalist model than in the traditional model. While reassuring, it is important to remember that the patient experience does not begin at admission or end at discharge, and a more patient-centered analysis might take into account the outpatient providers too (ie, those seeing the patient before admission and after discharge), and would probably show that the hospitalist model indeed leads to more care fragmentation. After all, there are at least 2 providers involved in every patient's care when a hospitalist model is used, whereas a large subset of patients cared for by PCPs would have only 1 provider involved.

While not the primary focus of the analysis, Fletcher and colleagues ${ }^{4}$ identified additional predictors of inpatient continuity of care. Higher socioeconomic class and white race were associated with lower continuity. This suggests that care fragmentation is not a feature of inferior, or at least cheap, care. In keeping with this observation, there was also enormous geographic variation in inpatient care continuity, marked by greater fragmentation of care in the New England and the mid-Atlantic regions than in other areas of the country, and more fragmentation in larger hospitals serving heavily populated metropolitan areas. This pattern is strikingly similar to the cost-of-care patterns observed by the Dartmouth Atlas researchers. ${ }^{5,6}$ Densely populated areas tend to have more specialists per capita and also tend to deliver more expensive care-without demonstrably higher quality. In parallel, it is easy to see how care fragmentation might increase length-of-stay ${ }^{7}$ and lead to excessive diagnostic testing and consultation. More cooks in the kitchen might make costlier stew.

How hospitalists tackle the issue of inpatient continuity is not only a matter of quality of care, but also a matter of job sustainability. The simple way to maximize continuity-working many consecutive dayscan lead to burnout if taken too far. But there are 
creative ways to assign admissions that maximize continuity for the average inpatient while allowing providers needed time off. The CICLE initiative (Creating Incentives and Continuity Leading to Efficiency in hospital medicine) at the Johns Hopkins Bayview Medical Center, for instance, assigns physicians to 4day cycles of clinical work; the first day of the cycle (a long-call day) involves admitting a large number of patients during a busy shift, with no new patients admitted on the remaining days of the cycle. Thus, all patients whose length of stay is less than 5 days will have a single attending-of-record. Not only does this model increase continuity, it also incentivizes providers to augment throughput: more discharged patients on Tuesday means fewer patients to see on Wednesday, without any expectation to backfill. Other less aggressive but similar approaches are used elsewhere, such as exempting hospitalists from accepting new patients on the last 1 or 2 of the consecutive days they work. We eagerly await data on the impact of these programs on quality of care, patient satisfaction, and provider satisfaction.

The impact of other providers and staff cannot be ignored. While the most important handoff in many cases may indeed be between the PCP and attending hospitalist tasked with coordinating the overall care of the patient, for some patients, there may be a specialist who has known the patient for years who is driving the plan of care. For patients with severe chronic illnesses, such as end-stage renal disease or asthma, a well-structured specialty clinic may even serve as a patient-centered medical home. ${ }^{8}$ And the current inpatient team includes night coverage physicians (whether moonlighters, house staff, or covering hospitalists), and an ever-increasing number of nonphysicians who play a critical role in hospital care (non-physician providers, nurses, social workers, pharmacists, case managers, physical therapists, and others). While it is tempting to focus on the attending physician as the main driver of healthcare quality, continuity, and the inpatient experience, this is an oversimplification.

If there is a take-home message, it is probably that most hospitalized patients will be cared for by multiple providers and a team of non-physicians. The "Marcus Welby" practice model may not be completely dead, but if "Dr. Welby" were still in practice, it would be a safe bet that he would be slower at computerized order entry than the average intern, that financial pressures would make it hard for him to attend to his hospitalized patients, and that he probably would have turned over much of his inpatient practice to the physicians and non-physician caregivers who make the hospital their primary workplace. ${ }^{9}$ Going forward, research should examine ways to optimize care coordination under the hospitalist model, ${ }^{10-13}$ rather than comparing it to the traditional model of inpatient care. The ingredients for success include coordinated care by a committee of caregivers, effective handoffs (throughout hospitalization and at discharge), ${ }^{12,14}$ focused and deliberate multidisciplinary communication, and effective patient education, ${ }^{15}$ regardless of the attending-du-jour.

Disclosure: Nothing to report.

\section{References}

1. Coffman J, Rundall TG. The impact of hospitalists on the cost and quality of inpatient care in the United States: a research synthesis. Med Care Res Rev. 2005;62:379-406.

2. Auerbach AD, Aronson MD, Davis RB, Phillips RS. How physicians perceive hospitalist services after implementation: anticipation vs reality. Arch Intern Med. 2003;163:2330-2336.

3. Lindenauer PK, Rothberg MB, Pekow PS, Kenwood C, Benjamin EM, Auerbach AD. Outcomes of care by hospitalists, general internists, and family physicians. N Engl J Med. 2007;357:2589-2600.

4. Fletcher K, Sharma G, Dong Z, Yong-fang K, Goodwin J. Trends in inpatient continuity of care for a cohort of Medicare patients, 19962006. J Hosp Med. 2011;6:438-444.

5. Fisher ES, Wennberg DE, Stukel TA, Gottlieb DJ, Lucas FL, Pinder EL. The implications of regional variations in Medicare spending. Part 2: health outcomes and satisfaction with care. Ann Intern Med. 2003;138:288-298.

6. Fisher ES, Wennberg DE, Stukel TA, Gottlieb DJ, Lucas FL, Pinder EL. The implications of regional variations in Medicare spending. Part 1: the content, quality, and accessibility of care. Ann Intern Med. 2003;138:273-287.

7. Epstein K, Juarez E, Epstein A, Loya K, Singer A. The impact of fragmentation of hospitalist care on length of stay. J Hosp Med. 2010;5: 335-338

8. Kirschner N, Barr MS. Specialists/subspecialists and the patient-centered medical home. Chest. 2010;137:200-204.

9. Meltzer DO, Chung JW. U.S. trends in hospitalization and generalist physician workforce and the emergence of hospitalists. J Gen Intern Med. 2010;25:453-459.

10. Hinami K, Farnan JM, Meltzer DO, Arora VM. Understanding communication during hospitalist service changes: a mixed methods study. J Hosp Med. 2009;4:535-540.

11. Halasyamani L, Kripalani S, Coleman E, et al. Transition of care for hospitalized elderly patients-development of a discharge checklist for hospitalists. J Hosp Med. 2006;1:354-360.

12. Arora VM, Manjarrez E, Dressler DD, Basaviah P, Halasyamani L, Kripalani S. Hospitalist handoffs: a systematic review and task force recommendations. J Hosp Med. 2009;4:433-440.

13. Wachter RM. The hospitalist field turns 15: new opportunities and challenges. J Hosp Med. 2011;6:E1-E4.

14. Snow V, Beck D, Budnitz T, et al. Transitions of Care Consensus Policy Statement American College of Physicians-Society of General Internal Medicine-Society of Hospital Medicine-American Geriatrics Society-American College of Emergency Physicians-Society of Academic Emergency Medicine. J Gen Intern Med. 2009;24:971-976.

15. Jack BW, Chetty VK, Anthony D, et al. A reengineered hospital discharge program to decrease rehospitalization: a randomized trial. Ann Intern Med. 2009;150:178-187. 